Poi nt transf or mat i ons appl i ed to densi ty-f unct i onal cal cul at i ons

\begin{tabular}{|l|l|}
\hline 著者 & KOGA Toshi kat su \\
\hline $\begin{array}{l}\text { j our nal or } \\
\text { publ i cat i on ti tl e }\end{array}$ & $\begin{array}{l}\text { Physi cal revi ew. Ser. 3. A, At om c, nol ecul ar, } \\
\text { and opt i cal physi cs }\end{array}$ \\
\hline vol une & 93 \\
\hline number & 5 \\
\hline page $r$ ange & $3720-3722$ \\
\hline year & $1990-02-01$ \\
\hline URL & ht t p: //hdl . handl e. net /10258/862 \\
\hline
\end{tabular}




\title{
Point transformations applied to density-functional calculations
}

\author{
Toshikatsu Koga \\ Department of Applied Chemistry and Department of Applied Science for Energy, Muroran Institute of Technology, \\ Muroran, Hokkaido 050, Japan
}

(Received 3 October 1989)

\begin{abstract}
The method of point transformations enables us to generate an unknown wave function $\Psi_{\rho}\left(\left\{\mathbf{r}_{k}\right\}\right)$ from a given electron density $\rho(\mathbf{r})$ based on a reference wave function $\Psi_{0}\left(\left\{\mathbf{r}_{k}\right\}\right)$ initially chosen for the system. The generated wave function $\Psi_{\rho}\left(\left\{\mathbf{r}_{k}\right\}\right)$ can be used to evaluate the energy $E_{\rho}$ associated with the given density $\rho(\mathbf{r})$. This procedure constitutes a rigorous density-functional theory, if the density $\rho(\mathbf{r})$ and reference function $\Psi_{0}\left(\left\{\mathbf{r}_{k}\right\}\right)$ are varied. Within the Hartree-Fock framework numerical applications are presented for the ground-state helium atom and its analogs both in position and momentum spaces. Simple position and momentum densities are reported that well reproduce the Hartree-Fock limit energy. A few calculations beyond the Hartree-Fock approximation are also given.
\end{abstract}

\section{INTRODUCTION}

Recently, Kryachko and co-workers proposed ${ }^{1-4}$ an application of the method of point transformations (see, e.g., Ref. 5 for a review) to the electron density function $\rho(\mathbf{r})$ of an $N$-electron system. They first choose an appropriate reference density $\rho_{0}(\mathbf{r})$ whose parent wave function $\Psi_{0}\left(\left\{\mathbf{r}_{k}\right\}\right)$ is known, and construct for a given $\rho(\mathbf{r})$ a unique single-electron transformation $T$ such that $\rho(\mathbf{r})=T \rho_{0}(\mathbf{r})$. Then the direct product $T^{N}$ of the singleelectron transformation $T$ is applied to the reference wave function $\Psi_{0}\left(\left\{\mathbf{r}_{k}\right\}\right)$ to obtain a wave function $\Psi_{\rho}\left(\left\{\mathbf{r}_{k}\right\}\right)$ corresponding to the given density $\rho(\mathbf{r})$. Once the wave function $\Psi_{\rho}\left(\left\{r_{k}\right\}\right)$ is generated, the construction $^{6}$ of density matrices $\Gamma_{\rho}^{(n)}\left(\mathbf{r}_{1}^{\prime} \mathbf{r}_{2}^{\prime} \cdots \mathbf{r}_{n}^{\prime} \mid \mathbf{r}_{1} \mathbf{r}_{2} \cdots \mathbf{r}_{n}\right)$ is straightforward. Kryachko and co-workers ${ }^{1-4}$ have used this procedure mainly for the study of density-functional formalism. An analogous theory has been developed ${ }^{7}$ in momentum space, and the mapping relation between the momentum density $\Pi(\mathbf{p})$ and the position density $\rho(\mathbf{r})$ is discussed as well as the momentum-space density functional of the energy. However, these studies are rather formal, and hence the practical utility and the numerical reliability of the proposed procedure have remained unclear.

Very recently, Koga, Yamamoto, and Kryachko have examined ${ }^{8}$ the density mapping between momentum and position spaces for the ground state of the helium atom, using the proposed method of point transformations. From a given momentum density $\Pi(p)$, they have been able to reproduce the position density $\rho(\mathbf{r})$ and the position moment $\left\langle r^{n}\right\rangle(-2 \leq n \leq 4)$ within $2 \%$ error. The inverse procedure has given the momentum density $\Pi(\mathbf{p})$ and the momentum moment $\left\langle p^{n}\right\rangle$ within $1 \%$ error, and the accuracy of the proposed method has been found to be quite satisfactory.

In the present paper, we apply the method of point transformations to the density-functional calculation of the ground-state energy of the heliumlike atoms within and beyond the Hartree-Fock framework. In Sec. II, the theory of point transformations is outlined for the generation of a wave function from a given density. A special aspect of the method for the heliumlike ground state is discussed that the generated wave function is independent of the reference quantities within the Hartree-Fock theory. In Sec. III, the results of density-functional calculations are presented for the ground-state heliumlike atoms both in position and momentum spaces. The energy-optimized densities and associated moments are compared with those obtained from the ordinary Hartree-Fock calculations. Simple position and momentum densities which well reproduce the near HartreeFock results are reported. Illustrative treatment beyond the Hartree-Fock approximation is also presented. Atomic units are used throughout this paper.

\section{MAPPING FROM DENSITY TO WAVE FUNCTION AND DENSITY MATRICES}

For an $N$-electron system, we assume that a prototypical wave function $\Psi_{0}\left(\left\{\mathbf{r}_{k}\right\}\right)$ and the associated density $\rho_{0}(\mathbf{r})$ are known as a reference. To generate a wave function $\Psi_{\rho}\left(\left\{\mathbf{r}_{k}\right\}\right)$ corresponding to a given density $\rho(\mathbf{r})$, we first establish a single-electron transformation

$$
\begin{aligned}
\rho(\mathbf{r}) & =T \rho_{0}(\mathbf{r}) \\
& =J(\mathbf{s} / \mathbf{r}) \rho_{0}(\mathbf{s}),
\end{aligned}
$$

where $J(s / r)$ is the Jacobian for the variable transformation $\mathbf{s}=\mathbf{s}(\mathbf{r})$ and guarantees the relation

$$
J(\mathbf{s} / \mathbf{r}) d \mathbf{r}=d \mathbf{s} .
$$

If we use the spherical coordinates $(r, \Omega)$ and $(s, \omega)$ for the vectors $\mathbf{r}$ and $\mathbf{s}$, respectively, and if we consider the point transformation along a definite angular direction, Eq. (1a) is rewritten as ${ }^{1-4}$

$$
\rho(r, \Omega)=J(\mathbf{s} / \mathbf{r}) \rho_{0}(s, \Omega),
$$


where

$$
\begin{aligned}
& J(s / \mathbf{r})=(s / r)^{2}(d s / d r), \\
& s=s(r, \Omega) .
\end{aligned}
$$

For the given set of $\rho_{0}(\mathbf{r})$ and $\rho(\mathbf{r})$, the radial point transformation $s=s(r, \Omega)$ is obtained ${ }^{7}$ as a solution of either the differential equation

$$
d[s(r, \Omega)]^{3} / d r=3 r^{2}\left[\rho(r, \Omega) / \rho_{0}(s(r, \Omega), \Omega)\right],
$$

or the integral equation

$$
\int_{0}^{r} \rho(x, \Omega) x^{2} d x-\int_{0}^{s(r, \Omega)} \rho_{0}(y, \Omega) y^{2} d y=0 .
$$

When the system has spherical symmetry, the angular dependence vanishes in Eqs. (2a)-(3b).

We next apply the direct product $T^{N}$ of the singleelectron transformation $T$ thus determined to the reference wave function $\Psi_{0}\left(\left\{r_{k}\right\}\right)$ :

$$
\begin{aligned}
& \Psi_{\rho}\left(\left\{\mathbf{r}_{k}\right\}\right)=T^{N} \Psi_{0}\left(\left\{\mathbf{r}_{k}\right\}\right) \\
&\left.=\int \prod_{k=1}^{N} J\left(\mathbf{s}_{k} / \mathbf{r}_{k}\right)^{1 / 2}\right] \Psi_{0}\left(\left\{\mathbf{s}_{k}\right\}\right), \\
& \mathbf{s}_{k}=\mathbf{s}\left(\mathbf{r}_{k}\right) \text { or } \mathbf{s}_{k}=\left(s_{k}, \Omega_{k}\right), \quad s_{k}=s\left(r_{k}, \Omega_{k}\right) .
\end{aligned}
$$

The generated wave function $\Psi_{\rho}\left(\left\{\mathbf{r}_{k}\right\}\right)$ has the electron density exactly the same as the given density $\rho(\mathbf{r})$. Clearly, the transformation $T$ and therefore the generated wave function $\Psi_{\rho}$ are functionals of the reference wave function $\Psi_{0}$.

Once the wave function $\Psi_{\rho}\left(\left\{\mathbf{r}_{k}\right\}\right)$ is generated, we can construct various density matrices

$$
\Gamma_{\rho}^{(n)}\left(\mathbf{r}_{1}^{\prime} \cdots \mathbf{r}_{n}^{\prime} \mid \mathbf{r}_{1} \cdots \mathbf{r}_{n}\right)
$$

and their momentum-space counterparts

$$
\Pi_{\rho}^{(n)}\left(\mathbf{p}_{1}^{\prime} \cdots \mathbf{p}_{n}^{\prime} \mid \mathbf{p}_{1} \cdots \mathbf{p}_{n}\right)
$$

straightforwardly, all of which are consistent with the given density $\rho(\mathbf{r})$. For the calculation of nonrelativistic energies, the three types of reduced density matrices are sufficient. $^{6}$ They are given by

$$
\begin{aligned}
& \Gamma_{\rho}\left(\mathbf{r}_{1}, \mathbf{r}_{2}\right)=J\left(\mathbf{s}_{1} / \mathbf{r}_{1}\right) J\left(\mathbf{s}_{2} / \mathbf{r}_{2}\right) \Gamma_{0}\left(\mathbf{s}_{1}, \mathbf{s}_{2}\right), \\
& \gamma_{\rho}\left(\mathbf{r}_{1}^{\prime} \mid \mathbf{r}_{1}\right)=\left[J\left(\mathbf{s}_{1}^{\prime} / \mathbf{r}_{1}^{\prime}\right) J\left(\mathbf{s}_{1} / \mathbf{r}_{1}\right)\right]^{1 / 2} \gamma_{0}\left(\mathbf{s}_{1}^{\prime} \mid \mathbf{s}_{1}\right),
\end{aligned}
$$

apart from $\rho(\mathbf{r})$ which is assumed to be given. So long as the energy is concerned, we therefore have no need to know the reference wave function $\Psi_{0}\left(\left\{\mathbf{r}_{k}\right\}\right)$, but the reference two-matrix $\Gamma_{0}\left(\mathbf{r}_{1}^{\prime} \mathbf{r}_{2}^{\prime} \mid \mathbf{r}_{1} \mathbf{r}_{2}\right)$ suffices for the generation of the required quantities.

The above procedure reveals an interesting aspect for the ground state of heliumlike atoms. Within the Hartree-Fock approximation, (the spatial part of) a reference wave function for this state is expressible as

$$
\Psi_{0}\left(\mathbf{r}_{1}, \mathbf{r}_{2}\right)=\psi_{0}\left(\mathbf{r}_{1}\right) \psi_{0}\left(\mathbf{r}_{2}\right),
$$

where we assume that $\psi_{0}(\mathbf{r})$ is a real function. For a given density $\rho(r)$, we then have

$$
J(\mathbf{s} / \mathbf{r})=\rho(\mathbf{r}) / \rho_{0}(\mathbf{s})=\rho(\mathbf{r}) / 2\left[\psi_{0}(\mathbf{s})\right]^{2},
$$

and hence

$$
\Psi_{\rho}\left(\mathbf{r}_{1}, \mathbf{r}_{2}\right)=\left[\rho\left(\mathbf{r}_{1}\right) \rho\left(\mathbf{r}_{2}\right)\right]^{1 / 2} / 2,
$$

where the factor of 2 arises from the normalization of the electron densities to two electrons. Equation (6c) implies the following important fact: The generated wave function $\Psi_{\rho}$ is completely free from the reference wave function in this particular case. This point will be fully utilized in Sec. III.

If we are working in momentum space, we first assume an appropriate reference momentum wave function $\Phi_{0}\left(\left\{p_{k}\right\}\right)$ and the associated momentum density $\Pi_{0}(p)$. Then the exactly analogous discussion given above in position space holds for a given momentum density $\Pi(p)$.

\section{DENSITY-FUNCTIONAL CALCULATIONS FOR THE GROUND STATE OF HELIUMLIKE ATOMS}

\section{A. Within Hartree-Fock approximation}

\section{Position space}

We have first assumed a density function $\rho(\mathbf{r} ; a, b, c, \ldots)$ which has adjustable parameters $a, b, c, \ldots$. The corresponding Hartree-Fock wave function $\Psi_{\rho}\left(\mathbf{r}_{1}, \mathbf{r}_{2} ; a, b, c, \ldots\right)$ has been generated according to the procedure described in Sec. II. Then the associated energy $E_{\rho}(a, b, c, \ldots)$ has been evaluated numerically using the Romberg integration method, ${ }^{9}$ and the parameters $a, b, c, \ldots$ have been optimized to minimize the energy $E_{\rho}$ using the Powell method of conjugate directions. ${ }^{10}$

For the helium atom, we have examined several simple density functions constructed from exponential functions. The results are summarized in Table $I$. It is seen that the five-parameter function

$\rho(\mathbf{r})=\exp (-a r)+b \exp (-c r)+d \exp (-e r), \quad r=|\mathbf{r}|$

well reproduces $\left(E_{\rho}=-2.8616799\right)$ the near HartreeFock limit energy ${ }^{11-14}-2.8616800$. Equation (7) represents a linear combination of three hydrogenic $1 s$ densities and is considerably simpler than that derived ${ }^{17}$ from the near Hartree-Fock linear combination of atomic orbitals wave function due to Clementi and Roetti. ${ }^{12}$ The position moments $\left\langle r^{n}\right\rangle(-2 \leq n \leq 4)$ associated with this density [Eq. (7)] are given in Table II in comparison with the near Hartree-Fock limit results. ${ }^{17}$ The agreement is excellent in spite of the simplicity of the present density.

We have also generated numerically the momentum wave function $\Phi_{\rho}\left(\mathbf{p}_{1}, \mathbf{p}_{2}\right)$ and the momentum density $\Pi_{\rho}(\mathbf{p})$ corresponding to the given density $\rho(\mathbf{r})$ [Eq. (7)] through the Fourier transformation of $\Psi_{\rho}\left(r_{1}, r_{2}\right)$. Since the generated position wave function $\Psi_{\rho}$ is a function of the moduli $r_{1}$ and $r_{2}$ in the present case, the required transformation reduces to the Hankel transformation with the kernel $j_{0}(p r)$, i.e., the spherical Bessel function of the first kind. Therefore, we have used the logarithmic-scale-Fourier-Bessel-transform algorithm of Talman $^{18}$ for this purpose. In order to see the quality of 
TABLE I. Several position densities examined for the helium atom within the Hartree-Fock approximation. The density functions are not normalized.

\begin{tabular}{|c|c|c|}
\hline $\begin{array}{l}\text { No. of } \\
\text { parameters }\end{array}$ & $\begin{array}{l}\text { Position density function } \\
\text { (optimum parameters) }\end{array}$ & Energy \\
\hline 1 & $\begin{array}{l}\exp (-a r) \\
(a=3.3750)\end{array}$ & $-2.8476562^{\mathrm{a}}$ \\
\hline 2 & $\begin{array}{l}r^{a} \exp (-b r) \\
(a=-0.0900, b=3.2232)\end{array}$ & -2.8542175 \\
\hline 2 & $\begin{array}{l}\exp \left(-a r^{b}\right) \\
(a=3.6510, b=0.8966)\end{array}$ & $-2.8610734^{b}$ \\
\hline 3 & $\begin{array}{l}r^{a} \exp \left(-b r^{c}\right) \\
(a=0.0420, b=3.8005, c=0.8727)\end{array}$ & $-2.8614782^{b}$ \\
\hline 3 & $\begin{array}{l}\exp (-a r)+b \exp (-c r) \\
(a=2.9424, b=1.3226, c=4.7464)\end{array}$ & -2.8616517 \\
\hline 4 & $r^{a}[\exp (-b r)+c \exp (-d r)]$ & -2.8616566 \\
\hline 4 & $\begin{array}{l}(a=-0.0039, b=2.9337, c=1.3383, d=4.6970) \\
\exp \left(-a r^{b}\right)+c \exp \left(-d r^{b}\right) \\
(a=3.0073, b=0.9841, c=1.2326, d=4.6606)\end{array}$ & -2.8616714 \\
\hline \multirow[t]{2}{*}{5} & $\begin{array}{l}\exp (-a r)+b \exp (-c r)+d \exp (-e r) \\
(a=2.8024, b=1.4190, c=3.5822 \\
d=1.5099, e=5.2275)\end{array}$ & -2.8616799 \\
\hline & Near Hartree-Fock limit & $-2.8616800^{c}$ \\
\hline
\end{tabular}

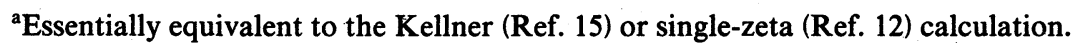

${ }^{b}$ Essentially equivalent to the generalized STO calculation (Ref. 16).

${ }^{\mathrm{c}}$ References $11-14$.

the generated momentum density $\Pi_{\rho}(\mathbf{p})$, we have calculated the momentum moments $\left\langle p^{n}\right\rangle(-2 \leq n \leq 4)$. In Table II, the results are tabulated and compared with the near Hartree-Fock limit values. The agreement is found to be quite satisfactory, though there remains some deviation in the fourth moment.

Since the position density in the form of Eq. (7) yields satisfactory results for the helium atom, we have applied the density of the same functional form to several twoelectron atomic ions. The results are summarized in Table III, where we find that the near Hartree-Fock limit energies $^{11-14,20,21}$ are reproduced almost completely in all cases.

The present approach bears some resemblance to that discussed by $\mathrm{Hall}^{22}$ and ten $\mathrm{Hoor}^{23,24}$ for two-electron atoms. However, the essential differences are that (i) they have studied the point transformation at the level of wave functions and (ii) they have examined transformations resulting from simple analytical transformation functions $s=s(r)$ such as $s(r)=\left[(1+4 a r)^{1 / 2}-1\right] /(2 a)$, in which $a$ is a variational parameter.

\section{Momentum space}

An analogous study has been carried out in momentum space. Assuming a momentum density $\Pi(\mathbf{p} ; a, b, c, \ldots)$, we first construct the momentum wave function $\Phi_{\Pi}\left(\mathbf{p}_{1}, \mathbf{p}_{2} ; a, b, c, \ldots\right)$, from which we then generate the position wave function $\Psi_{\Pi I}\left(\mathbf{r}_{1}, \mathbf{r}_{2} ; a, b, c, \ldots\right)$ and the position density $\rho_{\Pi}(\mathbf{r} ; a, b, c, \ldots)$. The parameters $a, b, c, \ldots$ embedded in the momentum density $\Pi$ are so determined as to minimize the energy $E_{\mathrm{II}}$ associated with the gen-

TABLE II. Comparison of the position and momentum moments for the helium atom obtained from the five-parameter position density [Eq. (7)] with the near Hartree-Fock limit values.

\begin{tabular}{|c|c|c|c|c|c|}
\hline & $\begin{array}{c}\text { Position n } \\
\text { Present } \\
\text { [Eq. (7)] }\end{array}$ & $\begin{array}{c}\text { Near } \\
\text { Hartree-Fock }^{\mathrm{a}}\end{array}$ & & $\begin{array}{c}\text { Momentum } \\
\text { Present } \\
\text { [Eq. (7)] }\end{array}$ & $\begin{array}{l}\text { Near } \\
\text { Hartree-Fock }\end{array}$ \\
\hline$\left\langle r^{-2}\right\rangle$ & 5.9959 & 5.996 & $\left\langle p^{-2}\right\rangle$ & 2.0461 & 2.0447 \\
\hline$\left\langle r^{-1}\right\rangle$ & 1.6873 & 1.68731 & $\left\langle p^{-1}\right\rangle$ & 1.0705 & 1.0703 \\
\hline$\langle r\rangle$ & 0.9273 & 0.92724 & $\langle p\rangle$ & 1.3995 & 1.3995 \\
\hline$\left\langle r^{2}\right\rangle$ & 1.1849 & 1.18464 & $\left\langle p^{2}\right\rangle$ & 2.8616 & 2.8618 \\
\hline$\left\langle r^{3}\right\rangle$ & 1.9407 & 1.93977 & $\left\langle p^{3}\right\rangle$ & 8.9961 & 8.9960 \\
\hline$\left\langle r^{4}\right\rangle$ & 3.8882 & 3.8838 & $\left\langle p^{4}\right\rangle$ & 52.9 & 52.841 \\
\hline
\end{tabular}

${ }^{\text {a }}$ Reference 17.

${ }^{\mathrm{b} C a l c u l a t e d}$ from the Clementi-Roetti wave function (Ref. 12). See also Ref. 19. 
TABLE III. Position-space density-functional calculations within the Hartree-Fock approximation for the ground state of several heliumlike atoms. The functional form of the position density is given by Eq. (7).

\begin{tabular}{|c|c|c|c|}
\hline Atom & \multicolumn{2}{|c|}{ Position density functional [Eq. (7)] } & $\begin{array}{c}\text { Near } \\
\text { Hartree-Fock } \\
\text { limit energy }\end{array}$ \\
\hline $\mathbf{H}^{-}$ & $\begin{array}{c}a=0.8106, b=8.2572, c=1.3857 \\
d=13.704, e=2.4380\end{array}$ & -0.4879262 & $-0.4879297^{a}$ \\
\hline $\mathrm{He}$ & $\begin{array}{c}a=2.8024, b=1.4190, c=3.5822 \\
d=1.5099, e=5.2275\end{array}$ & -2.8616799 & $-2.8616800^{\mathrm{a}, \mathrm{b}}$ \\
\hline $\mathrm{Li}^{+}$ & $\begin{array}{c}a=4.7577, b=0.9456, c=5.4893 \\
d=0.9931, e=7.8069\end{array}$ & -7.2364148 & $-7.2364152^{\mathrm{a}}$ \\
\hline $\mathrm{Be}^{2+}$ & $\begin{array}{c}a=6.7564, b=0.6527, c=7.5102 \\
d=0.6669, e=10.448\end{array}$ & -13.611299 & $-13.611299^{a}$ \\
\hline $\mathbf{B}^{3+}$ & $\begin{array}{c}a=8.7828, b=0.3945, c=9.6049 \\
d=0.4661, e=13.079\end{array}$ & -21.986233 & $-21.98623^{c}$ \\
\hline
\end{tabular}

${ }^{a}$ Reference 14

${ }^{\mathrm{b}}$ Reference 13.

${ }^{\mathrm{c}}$ Reference 21 .

erated wave functions $\Phi_{\Pi}$ and $\Psi_{\Pi}$.

As trial momentum densities, we have examined the hydrogenic $1 s$ density $\left(p^{2}+\zeta^{2}\right)^{-4}$ and its several modifications. The results of the energy minimization are given in Table IV for the helium atom. The fiveparameter momentum density

$\Pi(\mathbf{p})=\left(p^{2}+a\right)^{-4}+b\left(p^{2}+c\right)^{-4}+d\left(p^{2}+e\right)^{-4}, \quad p=|\mathbf{p}|$,

has given the best result $E_{\Pi}=-2.8616796$ in the present study. Similar to the position density (7), the momentum density (8) is also a linear combination of three hydrogenic $1 s$ densities in momentum space.

The position moments $\left\langle r^{n}\right\rangle$ and the momentum moments $\left\langle p^{n}\right\rangle(-2 \leq n \leq 4)$ accompanied with the momentum density (8) are compared in Table $\mathrm{V}$ with the corresponding near Hartree-Fock limit values. The overall agreement is good, though the deviation becomes larger as $|n|$ increases.

The applications of the momentum density (8) to the other heliumlike atoms are presented in Table VI. The agreement with the known Hartree-Fock limit ener$\mathrm{gy}^{11-14,20,21}$ is excellent as has been the case of the posi-

TABLE IV. Several momentum densities examined for the helium atom within the Hartree-Fock approximation. The density functions are not normalized.

\begin{tabular}{|c|c|c|}
\hline $\begin{array}{l}\text { No. of } \\
\text { parameters }\end{array}$ & $\begin{array}{l}\text { Momentum density function } \\
\text { (optimum parameters) }\end{array}$ & Energy \\
\hline 1 & $\begin{array}{l}\left(p^{2}+a\right)^{-4} \\
(a=2.8477)\end{array}$ & $-2.8476562^{2}$ \\
\hline 2 & $\begin{array}{l}\left(p^{2}+a\right)^{-b} \\
(a=2.3161, b=3.7154)\end{array}$ & -2.8585127 \\
\hline 2 & $\left(p^{a}+b\right)^{-4}$ & -2.8601445 \\
\hline 3 & $\begin{array}{l}(a=1.8840, b=2.2810) \\
\left(p^{a}+b\right)^{-c} \\
(a=1.7145, b=2.3146, c=4.5571)\end{array}$ & -2.8611699 \\
\hline 3 & $\begin{array}{l}\left(p^{2}+a\right)^{-4}+b\left(p^{2}+c\right)^{-4} \\
(a=2.2233, b=2.5554, c=6.4696)\end{array}$ & -2.8616483 \\
\hline 4 & $\begin{array}{l}p^{a}\left[\left(p^{2}+b\right)^{-4}+c\left(p^{2}+d\right)^{-4}\right] \\
(a=-0.0157, b=2.2602, c=2.5243, d=6.6779)\end{array}$ & -2.8616500 \\
\hline \multirow{3}{*}{5} & $\begin{array}{l}\left(p^{a}+b\right)^{-4}+c\left(p^{a}+d\right)^{-4} \\
(a=1.9866, b=2.1894, c=2.3026, d=6.0157)\end{array}$ & -2.8616653 \\
\hline & $\begin{array}{l}\left(p^{2}+a\right)^{-4}+b\left(p^{2}+e\right)^{-4}+d\left(p^{2}+e\right)^{-4} \\
(a=1.9764, b=2.1667, c=3.3754 \\
d=3.8428, e=8.0809)\end{array}$ & -2.8616796 \\
\hline & Near Hartree-Fock limit & $-2.8616800^{\mathrm{b}}$ \\
\hline
\end{tabular}

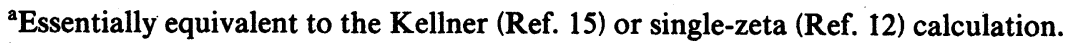

${ }^{\text {b}}$ References 11-14. 
TABLE V. Comparison of the position and momentum moments for the helium atom obtained from the five-parameter momentum density [Eq. (8)] with the near Hartree-Fock limit values.

\begin{tabular}{lccccc}
\hline \hline & $\begin{array}{c}\text { Position moments } \\
\text { Present } \\
\text { [Eq. (8)] }\end{array}$ & $\begin{array}{c}\text { Near } \\
\text { Hartree-Fock }\end{array}$ & & \multicolumn{3}{c}{$\begin{array}{c}\text { Momentum moments } \\
\text { Present } \\
\text { [Eq. (8)] }\end{array}$} & $\begin{array}{c}\text { Near } \\
\text { Hartree-Fock }\end{array}$ \\
\hline$\left\langle r^{-2}\right\rangle$ & 5.9964 & 5.996 & $\left\langle p^{-2}\right\rangle$ & 2.0464 & 2.0447 \\
$\left\langle r^{-1}\right\rangle$ & 1.6873 & 1.68731 & $\left\langle p^{-1}\right\rangle$ & 1.0705 & 1.0703 \\
$\langle r\rangle$ & 0.9273 & 0.92724 & $\langle p\rangle$ & 1.3995 & 1.3995 \\
$\left\langle r^{2}\right\rangle$ & 1.1848 & 1.18464 & $\left\langle p^{2}\right\rangle$ & 2.8617 & 2.8618 \\
$\left\langle r^{3}\right\rangle$ & 1.9407 & 1.93977 & $\left\langle p^{3}\right\rangle$ & 8.9973 & 8.9960 \\
$\left\langle r^{4}\right\rangle$ & 3.8884 & 3.8838 & $\left\langle p^{4}\right\rangle$ & 52.93 & 52.841 \\
\hline \hline
\end{tabular}

${ }^{\text {a }}$ Reference 17.

${ }^{b}$ Calculated from the Clementi-Roetti wave function (Ref. 12). See also Ref. 19.

tion density (7) (Table III).

Within the Hartree-Fock framework, the simple density functions, Eq. (7) in position space and Eq. (8) in momentum space, are concluded to be quite good approximations to the Hartree-Fock densities. When the two densities are compared, the position density (7) seems to be slightly superior to the momentum density (8).

\section{B. Beyond Hartree-Fock approximation}

The present study beyond the Hartree-Fock approximation is limited to position space for computational reason.

When we go beyond the Hartree-Fock framework, the energy $E$ is a functional of both the given density $\rho(\mathbf{r})$ and the reference wave function $\Psi_{0}\left(r_{1}, r_{2}\right)$,

$$
E=E\left[\rho, \Psi_{0}\right],
$$

i.e., the energy depends explicitly on the reference wave function $\Psi_{0}$ in contrast to the within Hartree-Fock treatment. In Eq. (9), $\Psi_{0}$ can be replaced with its second- order reduced density matrix $\Gamma_{0}\left(\mathbf{r}_{1}^{\prime} \mathbf{r}_{2}^{\prime} \mid \mathbf{r}_{1} \mathbf{r}_{2}\right)$ as has been mentioned. Then the calculus of the variations should follow formally. To simplify the matter, however, we fix the functional forms of $\rho(\mathbf{r})$ and $\Psi_{0}\left(r_{1}, r_{2}\right)$, and adjust the parameters involved.

For the density function $\rho(\mathbf{r})$, we adopt the function given by Eq. (7), which has been shown in Sec. III A to have a sufficient flexibility through the five parameters. As a reference system, we have chosen the Eckart wave function ${ }^{25}$ given by

$$
\begin{gathered}
\Psi_{0}\left(\mathbf{r}_{1}, \mathbf{r}_{2}\right)=\pi^{-1}(\alpha \beta)^{3 / 2}\left(2+2 S^{2}\right)^{1 / 2} \\
\times\left[\exp \left(-\alpha r_{1}-\beta r_{2}\right)\right. \\
\left.+\exp \left(-\beta r_{1}-\alpha r_{2}\right)\right], \\
S=8(\alpha \beta)^{3 / 2} /(\alpha+\beta)^{3},
\end{gathered}
$$

where $\alpha$ and $\beta$ are variational parameters. In the case of helium, for example, the optimum values are found to be $\alpha=2.183171$ and $\beta=1.188531$. The associated energy is

\begin{tabular}{|c|c|c|c|}
\hline \multirow[b]{2}{*}{ Atom } & \multicolumn{2}{|c|}{ Momentum density functional [Eq. (8)] } & \multirow{2}{*}{$\begin{array}{c}\text { Near } \\
\text { Hartree-Fock } \\
\text { limit energy }\end{array}$} \\
\hline & Optimum parameters & Energy & \\
\hline $\mathbf{H}^{-}$ & $\begin{array}{c}a=0.1749, b=17.934, c=0.5576 \\
d=63.510, e=1.8075\end{array}$ & -0.4879277 & $-0.4879297^{a}$ \\
\hline $\mathrm{He}$ & $\begin{array}{c}a=1.9764, b=2.1667, c=3.3754 \\
d=3.8428, e=8.0809\end{array}$ & -2.8616796 & $-2.8616800^{a, b}$ \\
\hline $\mathrm{Li}^{+}$ & $\begin{array}{c}a=5.5707, b=1.6548, c=7.6861 \\
d=2.3190, e=17.951\end{array}$ & -7.2364145 & $-7.2364152^{a}$ \\
\hline $\mathrm{Be}^{2+}$ & $\begin{array}{c}a=11.063, b=1.6113, c=14.003 \\
d=1.7010, e=31.979\end{array}$ & -13.611298 & $-13.611299^{a}$ \\
\hline $\mathbf{B}^{3+}$ & $\begin{array}{c}a=18.118, b=2.4857, c=21.870 \\
d=1.8250, e=49.882\end{array}$ & -21.986233 & $-21.98623^{c}$ \\
\hline
\end{tabular}
$E=-2.8756613$, which clearly shows that the reference

TABLE VI. Momentum-space density-functional calculations within the Hartree-Fock approximation for the ground state of several heliumlike atoms. The functional form of the momentum density is given by Eq. (8).

${ }^{\text {a Reference } 14 .}$

${ }^{\mathrm{b}}$ Reference 13.

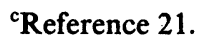


TABLE VII. Density-functional calculations beyond the Hartree-Fock approximation for the ground state of several heliumlike atoms. The Eckart wave function and density [Eqs. (10) and (11)] are used as reference system.

\begin{tabular}{|c|c|c|c|}
\hline \multirow[b]{2}{*}{ Atom } & \multicolumn{2}{|c|}{ Position density functional [Eq. (7)] } & \multirow{2}{*}{$\begin{array}{l}\text { Eckart } \\
\text { energy }\end{array}$} \\
\hline & Optimum parameters & Energy & \\
\hline $\mathbf{H}^{-}$ & $\begin{array}{c}a=1.0425, b=10.331, c=2.0879, d=0.0913 \\
e=0.4951, \alpha=1.0371, \beta=0.2836\end{array}$ & $\begin{array}{l}-0.5134555 \\
(0.0001526)^{\mathrm{a}}\end{array}$ & -0.5133029 \\
\hline $\mathrm{He}$ & $\begin{array}{c}a=2.7120, b=1.5012, c=3.5358, d=2.1147 \\
e=4.9848, \alpha=2.1926, \beta=1.1900\end{array}$ & $\begin{array}{r}-2.8768084 \\
(0.0011471)\end{array}$ & -2.8756613 \\
\hline $\mathrm{Li}^{+}$ & $\begin{array}{c}a=4.6691, b=1.1892, c=5.3512, d=1.4029 \\
e=7.5699, \alpha=3.3015, \beta=2.0789\end{array}$ & $\begin{array}{r}-7.2501004 \\
(0.0013525)\end{array}$ & -7.2487479 \\
\hline $\mathrm{Be}^{2+}$ & $\begin{array}{c}a=7.4391, b=1.4854, c=6.7092, d=1.1684 \\
e=10.225, \alpha=4.3960, \beta=2.9848\end{array}$ & $\begin{array}{r}-13.624400 \\
(0.001435)\end{array}$ & -13.622965 \\
\hline $\mathbf{B}^{3+}$ & $\begin{array}{c}a=8.7622, b=0.3273, c=9.7153, d=0.4800 \\
e=12.915, \alpha=5.4789, \beta=3.9012\end{array}$ & $\begin{array}{r}-21.999018 \\
(0.001479)\end{array}$ & -21.997539 \\
\hline
\end{tabular}

${ }^{a}$ Value in parentheses means the energy lowering from the ordinary Eckart calculation.

wave function is beyond the Hartree-Fock approximation. The Eckart wave function (10a) has the electron density

$$
\begin{array}{r}
\rho_{0}(\mathbf{r})=\pi^{-1}\left(1+S^{2}\right)^{-1}\left\{\alpha^{3} \exp (-2 \alpha r)+\beta^{3} \exp (-2 \beta r)\right. \\
\left.+2 S(\alpha \beta)^{3 / 2} \exp [-(\alpha+\beta) r]\right\} .
\end{array}
$$

Since we have fixed the functional forms of $\rho(\mathbf{r})$ and $\Psi_{0}\left(r_{1}, r_{2}\right)$ to those of Eqs. (7) and (10), respectively, the functional $E\left[\rho ; \Psi_{0}\right]$ now results in a function of seven adjustable parameters,

$$
E\left[\rho ; \Psi_{0}\right]=E(a, b, c, d, e ; \alpha, \beta),
$$

and therefore the variational problem reduces to the optimization of these parameters, We have carried out this simplified density-functional calculation using the methods described before.

Our final results are summarized in Table VII. In the case of the helium atom, we have obtained the energy -2.8768084 which is below the Eckart energy by 0.0011471 . A similar lowering of the energy is also observed in the table for the other two-electron atoms. It may be interesting to see that the energy from the present density-functional calculation is lower than the variational energy associated with the reference function (10). However, this is not surprising, since what we have done above can be considered as the variational calculation with a trial function

$$
\Psi\left(\mathbf{r}_{1}, \mathbf{r}_{2}\right)=\psi_{a}\left(r_{1}\right) \psi_{b}\left(r_{2}\right)+\psi_{b}\left(r_{1}\right) \psi_{a}\left(r_{2}\right),
$$

restricting its density to the form of Eq. (7). The two basis functions $\psi_{a}$ and $\psi_{b}$ involved in the trial function (13) are generated by the proposed point transformation from the simple exponential functions $\exp (-\alpha r)$ and $\exp (-\beta r)$, respectively, in a nonanalytical manner [compare Eqs. (10) and (13)]. Judging from the high flexibility of the density function (7) experienced in the within Hartree-Fock treatment (Sec. III A 1), we anticipate that the present energies tabulated in Table VII are close to the variational limits for the trial wave function (13).

\section{ACKNOWLEDGMENTS}

The author thanks Miss H. Gotoh for her computational assistance. Part of this study has been supported by a Grant-in-Aid for Scientific Research from the Ministry of Education of Japan.
${ }^{1}$ I. Zh. Petkov, M. V. Stoitsov, and E. S. Kryachko, Int. J. Quantum Chem. 29, 149 (1986).

${ }^{2}$ E. S. Kryachko, I. Zh. Petkov, and M. V. Stoitsov, Int. J. Quantum Chem. 32, 467 (1987).

${ }^{3}$ E. S. Kryachko, I. Zh. Petkov, and M. V. Stoitsov, Int. J. Quantum Chem. 32, 473 (1987).

${ }^{4}$ E. S. Kryachko and E. V. Ludena, Phys. Rev. A 35, 957 (1987).

${ }^{5}$ R. B. Wolf, Rev. Mex. Fis. 22, 45 (1973).

${ }^{6}$ P.-O. Löwdin, Phys. Rev. 97,1474 (1955).

${ }^{7}$ E. S. Kryachko and T. Koga, J. Chem. Phys. 91, 1108 (1989).

${ }^{8}$ T. Koga, Y. Yamamoto, and E. S. Kryachko, J. Chem. Phys. 91, 4758 (1989).

${ }^{9}$ See, for example, M. J. Maron, Numerical Analysis (Macmillan,
New York, 1982), p. 306ff.

${ }^{10}$ M. J. D. Powell, Comput. J. 7, 155 (1964).

${ }^{11}$ R. C. Raffenetti, J. Chem. Phys. 59, 5936 (1973).

${ }^{12}$ E. Clementi and C. Roetti, At. Data Nucl. Data Tables 14, 177 (1974).

13J. L. Gazquez and H. J. Silverstone, J. Chem. Phys. 67, 1887 (1977).

${ }^{14}$ C. C. J. Roothaan and G. A. Soukup, Int. J. Quantum Chem. 15, 449 (1979).

${ }^{15}$ G. W. Kellner, Z. Phys. 44, 91 (1927).

${ }^{16}$ R. H. Carrier and F. L. Pilar, J. Chem. Phys. 50, 2771 (1969).

${ }^{17}$ R. J. Boyd, Can. J. Phys. 55, 452 (1977).

18J. D. Talman, Comput. Phys. Commun. 30, 93 (1983). 
${ }^{19}$ S. R. Gadre, S. P. Gejii, and S. J. Chakravorty, At. Data Nuc. Data Tables 28, 477 (1983).

${ }^{20} \mathrm{C}$. F. Fischer, The Hartree-Fock Method for Atoms (Wiley, New York, 1977), p. 165.

${ }^{21}$ C. C. J. Roothaan, L. M. Sachs, and A. W. Weiss, Rev. Mod.
Phys. 32, 186 (1960).

${ }^{22}$ G. G. Hall, Proc. Phys. Soc. London 75, 575 (1960).

${ }^{23}$ M. J. ten Hoor, Int. J. Quantum Chem. 33, 467, 563 (1988).

${ }^{24}$ M. J. ten Hoor, J. Phys. B 22, L89 (1989).

${ }^{25}$ C. Eckart, Phys. Rev. 36, 878 (1930). 